\title{
Controlled deposition of MOFs by dip-coating in thin film nanocomposite membranes for organic solvent nanofiltration
}

Lilian Sarango, ${ }^{\mathrm{a}, \mathrm{b}}$ Lorena Paseta, ${ }^{\mathrm{a}}$ Marta Navarro, ${ }^{\mathrm{a}}$ Beatriz Zornoza ${ }^{\mathrm{a}}$ and Joaquín Coronas ${ }^{\mathrm{a}, *}$ ${ }^{a}$ Chemical and Environmental Engineering Department and Instituto de Nanociencia de Aragón (INA), Universidad de Zaragoza, 50018 Zaragoza, Spain ${ }^{\mathrm{b}}$ Electronics Engineering Career, Salesian Polytechnic University, Cuenca, Ecuador

*Corresponding author: coronas@unizar.es

\begin{abstract}
ZIF-8 and ZIF-67 particles, with sizes of $70 \pm 10 \mathrm{~nm}$ and $240 \pm 40 \mathrm{~nm}$, respectively, were deposited by dip-coating on top of polyimide $\mathrm{P} 84^{\circledR}$ asymmetric supports. In the best conditions, this gives rise to a MOF (metal-organic framework) monolayer which remains on the polyimide support during the interfacial polymerization of polyamide carried out to produce a thin film nanocomposite membrane for organic solvent nanofiltration (OSN). This method is simple, shorter and is environmentally friendly, since no excess MOF is lost during the interfacial polymerization, exhibiting good OSN results: dye rejection of $90 \%$ together with a high methanol permeance of $8.7 \mathrm{~L} \cdot \mathrm{m}^{-2} \cdot \mathrm{h}^{-1} \cdot \mathrm{bar}^{-1}$.
\end{abstract}

Keywords: Dip-coating; Thin film nanocomposite membrane; Organic solvent nanofiltration; Metal-organic framework; Zeolitic imidazolate framework. 


\section{Introduction}

In recent decades, organic solvent nanofiltration (OSN) with membranes has been very attractive due to its high separation efficiency and low equipment costs and energy consumption [1]. Materials that have been explored for the manufacture of high-performance composite membranes include carbon nanotubes [2], graphene [3], metal-organic frameworks (MOFs) [4], and covalent organic frameworks (COFs) [5], among others. MOFs are of great interest due to the exceptional properties of the design and functionalization of their pores, their high specific surface area and their easy synthesis, contributing to several membrane applications such as gas separation (GS) [6], reverse osmosis (RO) [7], pervaporation (PV) [8], nanofiltration (NF) $[4,9,10]$ and wastewater treatment [11]. Two types of polymer membranes can be found for NF and RO processes in terms of structure: integrally skinned asymmetric (ISA) membranes and thin film composite (TFC) membranes. ISA membranes consist of a selective thin layer supported on a more porous layer of the same material [12]. TFC membranes comprise a non-woven support at the bottom, an intermediate layer with an asymmetric porous structure, and a selective ultrathin film at the top. One of the most commonly used porous supports are polyimides (PI) owing to their unique physicochemical properties such as their resistance to high temperatures, radiation and chemical attack, their good mechanical strength and their superior insulation properties [13, 14]. The ultrathin selective layer is fabricated by the interfacial polymerization (IP) method [15]. This is carried out through a polycondensation reaction between two monomers present in two immiscible solutions: m-phenylediamine (MPD) aqueous and trimesoyl chloride (TMC) organic phases. As a consequence of this process, a thin barrier of polyamide (PA) layer is formed at the interface of the immiscible solutions giving rise to high solvent permeance and good retention of solutes $[16,17]$. 
With the aim of increasing the permeate flux without affecting the rejection, porous nanoparticles can be incorporated within the PA layer of TFC membranes producing the socalled thin film nanocomposite (TFN) membranes [18]. However, there remain several limitations such as the thickness of the skin layer [19, 20], and the difficulty of nanoparticle (MOF) positioning in the TFN [21]. In fact, conventional interfacial polymerization is poorly controllable because the reaction is completed in a few seconds, which represents a great challenge for forming a thin but defect-free skin layer. Therefore, the development of a method which initially controls the formation of a MOF monolayer avoiding aggregation is essential. In this context, Gevers et al. $[22,23]$ tested the dispersion of silica, carbon and zeolite (ZSM-5) inorganic fillers in a PDMS polymer matrix. Besides the enhancement of OSN, this strategy led to the reinforcement of the polymer and its anti-swelling properties. The incorporation of MOFs as fillers for the preparation of OSN membranes was first explored by Basu et al. [24] who incorporated $\mathrm{Cu}_{3}(\mathrm{BTC}) 2$, MIL-47, MIL-53(Al) and ZIF-8 dispersed into PDMS. This resulted in enhanced permeances with lower solute rejections compared with the pure PDMS/PI membrane (due to the poor adhesion of MOFs to PDMS). Campbell et al. [25] produced MMMs by the in situ growth (ISG) method of HKUST-1 crystals on preformed PI P $84{ }^{\circledR}$ membranes. These membranes demonstrated higher rejections in the treatment of styrene oligomer suspensions in acetone with a lower flux decline than those observed without the MOF. Sorribas et al. [4] prepared TFN membranes on PI P84 ${ }^{\circledR}$ asymmetric supports by dispersing several MOFs (ZIF-8, MIL-53(Al), NH2-MIL-53(Al) and MIL- 101(Cr)) in the organic phase before the IP reaction. These TFN membranes showed higher methanol and THF fluxes than commercial OSN membranes. Wang et al. [26] reported a similar procedure with ZIF-8 nanoparticles in the aqueous phase, in the organic phase, or in the two phases to produce a modified PA selective layer on asymmetric 
polysulfone (PSF). Wang et al. [27] used a layer-by-layer (LBL) method via ZIF-8 in-situ growth followed by IP to obtain TFN membranes containing ZIF-8.

These studies indicate that the development of OSN membranes faces challenges mainly in terms of the formation of defect-free skin layers with minimal thicknesses and of highly stable and selective operation. In this work, we propose a dip-coating method [28] for the controlled dispersion of MOF nanoparticles in TFN membranes. MOFs ZIF-8 [29] and ZIF-67 [30] (see Fig. 1) were chosen to create homogeneous monolayers on the surface of an asymmetric porous PI P84 ${ }^{\circledR}$ support after which the IP was carried out to produce PAbased TFN membranes. The membranes were applied in the OSN of methanol-dye solutions.

\section{Experimental Section}

\subsection{Materials}

Polyimide $\mathrm{P} 84^{\circledR}$ (PI - HP Polymer $\left.\mathrm{GmbH}\right), \mathrm{Zn}\left(\mathrm{NO}_{3}\right)_{2} \cdot 6 \mathrm{H}_{2} \mathrm{O}$ (99\% purity, Sigma Aldrich), $\mathrm{Co}\left(\mathrm{NO}_{3}\right)_{2} \cdot 6 \mathrm{H}_{2} \mathrm{O}$ (99\% purity, Sigma Aldrich), 2-methylimidazole (mIm, 99\%), methanol (99\% purity, Scharlab), m-phenylediamine (MPD - 99\%, Sigma Aldrich), DMF (99.5\%, Scharlab), dimethylsulfoxide (DMSO - synthesis grade, Scharlab), trymesoyl chloride (TMC - 98\%, Sigma Aldrich), hexane (99\%, Scharlab), isopropyl alcohol (IPA 99.5\%, Scharlab), hexanediamine (HDA - 98\%, Sigma Aldrich), polyethylenglycol (grade of PEG synthesis, Scharlab), Sunset Yellow (SY - 90\% dye content, Sigma Aldrich) and polypropylene (PP, Freudenberg Performance Materials) were used as received without further purification.

\subsection{Synthesis of MOFs}

ZIF-8 was synthesized based on the methodology reported elsewhere [31]. The following molar composition was used: $1 \mathrm{Zn}: 8 \mathrm{H}-\mathrm{MeIm}$ : $700 \mathrm{MeOH}$. $\mathrm{Zn}\left(\mathrm{NO}_{3}\right)_{2} \cdot 6 \mathrm{H}_{2} \mathrm{O}(0.47$ 
g) and 2-methylimidazole $(1 \mathrm{~g})$ were separately dissolved in $22.37 \mathrm{~mL}$ of methanol each. Then the solution of the ligand was poured on that of the salt and the resulting solution was stirred for $10 \mathrm{~min}$. This produced a white dispersion containing ZIF-8 nanosized crystals.

ZIF-67 was synthesized based on a previously reported molar composition [32], with an adaptation to $1 \mathrm{Co}: 8 \mathrm{H}-\mathrm{MeIm}: 700 \mathrm{MeOH}$ (from 1:4:700) $\mathrm{Co}\left(\mathrm{NO}_{3}\right)_{2} \cdot 6 \mathrm{H}_{2} \mathrm{O}(0.47 \mathrm{~g})$ and 2-methylimidazole $(1 \mathrm{~g})$ were separately dissolved in $22.67 \mathrm{~mL}$ of methanol each. Then the solution of the ligand was poured on that of the salt and the resulting solution was stirred for 10 min. A purple coloration indicated the formation of ZIF-67. The ZIF-8 and ZIF-67 suspensions were directly used for support dip coating without any modification.

\subsection{Preparation of porous substrates of $\mathrm{PB4}^{\circledR}$}

A polymer dope solution was prepared by dissolving 24 wt.\% polyimide $\left(\mathrm{P} 84^{\circledR}\right)$ in DMSO and stirring it overnight until complete dissolution. The viscous solution formed was allowed to stand until complete removal of the air bubbles. The dope solution was then cast on a non-woven PP sheet attached to a glass plate using the casting knife set Elcometer 4340 Automatic Film Applicator, at a thickness of $250 \mu \mathrm{m}$ and at a speed of $0.04 \mathrm{~m} \cdot \mathrm{s}^{-1}$. Immediately after casting, the polymer membrane was immersed in a distilled water bath at $20{ }^{\circ} \mathrm{C}$ where phase inversion occurred. After $10 \mathrm{~min}$, the coagulation bath was replaced with fresh distilled water and the substrates were left there for $1 \mathrm{~h}$.

The asymmetric P84 ${ }^{\circledR}$ supports were then immersed in two successive baths of IPA for $1 \mathrm{~h}$ each to remove any remains of water and DMSO [33]. Afterwards, a cross-linking process took place consisting of immersion in a bath of $120 \mathrm{~g} \cdot \mathrm{L}^{-1}$ of HDA in IPA for $16 \mathrm{~h}$ in order to increase the stability of the support in organic solvents. Four successive baths with IPA were then applied to remove traces of HDA from the supports. The supports were then 
immersed overnight in a PEG/IPA solution at a volume ratio of 3:2 to prevent pore collapse [9] and subsequently wiped with tissue paper to remove PEG excess.

\subsection{Preparation of homogeneous MOF@PI support by dip-coating technique}

Small disk supports of PI P84 $4^{\circledR}\left(12.0 \mathrm{~cm}^{2}\right)$ for characterization and large disk supports of the same material $\left(60.8 \mathrm{~cm}^{2}\right)$ for nanofiltration testing were dip coated with the suspensions of ZIF-8 and ZIF-67 freshly prepared (see step 2.2). After allowing $10 \mathrm{~s}$ for the entry into the suspension, the $\mathrm{P} 84^{\circledR}$ supports were dip-coated for $10 \mathrm{~min}$. The supports were then removed from the suspension for $10 \mathrm{~s}$ and dried overnight at $65^{\circ} \mathrm{C}$ giving rise to ZIFcoated supports, named MOF@PI_1L. The name MOF@PI_2L was used for twice coated supports, repeating the procedure described above with an intermediate drying of $20 \mathrm{~min}$ at $65{ }^{\circ} \mathrm{C}$. The large supports were placed in a glass filtration holder to facilitate the next step (see below). For the purpose of characterization, the remaining ZIF-8 and ZIF-67 suspensions were collected by repeated centrifugation (12000 rpm and $15 \mathrm{~min}$ ), rinsed with methanol three times, and dried overnight at $65^{\circ} \mathrm{C}$.

\subsection{Preparation of PA/MOF membrane by interfacial polymerization (IP)}

A polyamide (PA) thin layer was synthesized on ZIF-8@PI and ZIF-67@PI supports by interfacial polymerization (IP) to form PA/ZIF-8 and PA/ZIF-67 membranes, respectively. For this purpose, $30 \mathrm{~mL}$ of an aqueous solution of $2 \%(\mathrm{w} / \mathrm{v})$ MPD was poured over the membranes and after 2 min the excess solution was removed and the membrane was wiped with tissue paper. Then, $30 \mathrm{~mL}$ of a $0.1 \%(\mathrm{w} / \mathrm{v}) \mathrm{TMC}$ in hexane solution was added for $1 \mathrm{~min}$ to form the ultra-thin layer by IP. Before removing the excess solution, $10 \mathrm{~mL}$ of hexane was added to stop the reaction. Then, $10 \mathrm{~mL}$ of hexane was added to remove the unreacted TMC, and finally $10 \mathrm{~mL}$ of deionized water. TFC membranes were prepared 
following the same procedure as for the TFN membranes but without the MOF dip-coating. The formed PA/MOF membranes were stored in deionized water. Two post-treatments were applied to the TFC and TFN membranes. The first consisted of bath in a $50 \mathrm{~mL}$ DMF for 10 $\min$ at $20{ }^{\circ} \mathrm{C}$. The second consisted of filtering DMF for $10 \mathrm{~min}$ at a pressure of 20 bar at 25 ${ }^{\circ} \mathrm{C}$, right after $30 \mathrm{~min}$ of OSN (see below) [4, 6]. The PA/MOF TFN membranes prepared using different MOF@PI supports were named PA/MOF_xL, where $\mathrm{x}=1-2$ indicating the number of MOF dip-coatings carried out on the PI P84 $4^{\circledR}$ support.

\subsection{Characterization}

The surfaces and cross-sectional areas of the MOF@PI and PA/MOF samples were observed by scanning electron microscopy (SEM) using an Inspect-F (FEI) microscope operated at $15 \mathrm{~kW}$. Cross-sections of the membranes were prepared by freeze-fracturing after immersion in liquid $\mathrm{N}_{2}$ and subsequently coated with Pt. The MOF monolayer embedded between the PA and the PI support was also observed by means of a dual beam FIB/SEM (Nova NanoLab 200, FEI) equipped with an Everhart-Thornley Detector / Through Lens Detector (ETD/TLD) for secondary electron images. After the Au/Pt sputtering of the sample, the region of interest was protected via Pt deposition: a first electron deposition (at $5 \mathrm{kV}$ and $1.6 \mathrm{nA}$ ) of $300 \mathrm{~nm}$ followed by a second ion $\left(\mathrm{i}^{+}\right)$deposition (at $30 \mathrm{kV}$ and $0.3 \mathrm{nA}$ ) of $1 \mu \mathrm{m}$. SEM images were taken at $5 \mathrm{kV}$ with a Field Emission Gun (FEG) column, and a combined Ga-based $30 \mathrm{kV}$ ion beam ( $3 \mathrm{nA}$ for cross-section and 10-50 pA for cleaning crosssection) was used to cross-section the PA/MOF membrane. Energy-dispersive X-ray spectroscopy (EDX) was conducted for chemical analysis of the MOF layers added to the membranes. The average particle size was calculated using Image J 1.50i software by counting at least 250 particles. The Sturges method was used to construct the particle size histogram [34]. To facilitate the calculation of the MOF coating area on the PI P84 ${ }^{\circledR}$ porous 
support, a specific code for reading and processing SEM images was designed using MATLAB software. Powder X-ray diffraction (XRD) of MOF crystals was performed at room temperature using a D-Max Rigaku diffractometer with a copper anode and a graphite monochromator to select the $\mathrm{Cu} \operatorname{K} \alpha 1(\lambda=1.5406 \AA)$ radiation. The data were collected in the range $2 \theta=2.5-40^{\circ}$ and the scan speed was $0.01^{\circ} \mathrm{s}^{-1}$. Thermogravimetric analyses (TGA) of MOFs were performed using a Mettler Toledo TGA/SDTA 851e. Samples (10 mg) placed in $70 \mu \mathrm{L}$ alumina pans were heated in an air flow from 25 to $900{ }^{\circ} \mathrm{C}$ at a heating rate of 10 ${ }^{\circ} \mathrm{C} \cdot \mathrm{min}^{-1}$. Nitrogen adsorption-desorption measurements of MOF crystals were done in a Micrometrics Tristar 3000, and the Brunauer-Emmett-Teller (BET) method was used to calculate the corresponding specified surface areas. The experiments were performed at 77 $\mathrm{K}$ under variable relative pressure and the samples were degassed at $200{ }^{\circ} \mathrm{C}$ for $8 \mathrm{~h}$. FTIRATR (Fourier transform infrared spectroscopy attenuated total reflection) spectra were collected in a Bruker Vertex 70 spectrophotometer with a DTGS detector and diamond ATR Golden Gate. The wavenumber range measured in every case was between 500 and 4500 $\mathrm{cm}^{-1}$ with a resolution of $2 \mathrm{~cm}^{-1}$. This was used to study the MOF-polymer molecular interactions. To detect the presence of the MOFs in the thin film, the spectrum of the TFC membrane was subtracted from the spectra of the MOF@PI and PA/MOF membranes. The contact angle of the membranes was measured with a Krüss DSA 10 MK2 by dripping a water drop at three different places on each surface examined.

\subsection{Membrane Performance}

Nanofiltration experiments were performed in a dead-end membrane module (Sterlitech HP47501). The feed consisted of $250 \mathrm{~mL}$ of $20 \mathrm{mg} \cdot \mathrm{L}^{-1}$ of Sunset Yellow (SY, 450 $\left.\mathrm{g} \cdot \mathrm{mol}^{-1}\right)$ in methanol, which permeated through the membrane located at the bottom of the module. In order to adjust the membrane to the module, only $12 \mathrm{~cm}^{2}$ of the center of the 
membrane was selected as the effective area. The experiments were carried out at $20{ }^{\circ} \mathrm{C}$ and 20 bar of pressure and under constant stirring, being stronger working conditions than those normally used in OSN $[10,25]$. Once the process was stable, after approximately $30 \mathrm{~min}, 3$ $\mathrm{mL}$ of both permeate and residue were taken for further analysis. The membrane was then subjected to additional post-treatment by filtering DMF for 10 min at 20 bar and finally an extra nanofiltration experiment was carried out. The methanol in the samples was allowed to evaporate and was then replaced by $3 \mathrm{~mL}$ of deionized water. The absorbance of each sample was measured using a UV spectrometer (Jasco V-670 spectrophotometer) at the maximum absorbance wavelength of SY (480 nm). Afterwards, the concentration was obtained by means of a calibration curve. Permeation and rejection were calculated with equations S1-S3 in the ESI. The standard deviations of permeance and rejections of PA/ZIF-8 correspond to an average from 3-4 different membranes.

Finally, to corroborate the crystallinity preservation of the MOF after the IP and OSN processes, the IP was directly carried out (i.e. without the presence of the P84 $4^{\circledR}$ support) on both MOFs ZIF-8 and ZIF-67 at the same conditions described above with the difference that in this case both solutions (organic and aqueous with TMC and MPD, respectively) were mixed together under stirring. The MOF concentration in the formed unsupported PA/MOF composites was $0.6 \%(\mathrm{w} / \mathrm{v})$ instead of typical $0.2 \%(\mathrm{w} / \mathrm{v})[4]$. This was to favor the MOF detection by XRD. In addition, these composites were treated with a DMF bath during 10 min and put in the SY methanol feed solution during one day to simulate the OSN process.

\section{Results and discussion}

\subsection{MOFs characterization}


The ZIF-8 and ZIF-67 crystalline structures were confirmed by X-ray diffraction (see Fig. 2), both having the same sod type topology and thus similar patterns. Their thermal stability was determined by TGA (see Fig. S1 in the ESI). The adsorption/desorption isotherms exhibit the expected rapid increase in nitrogen adsorption at very low relative pressure and a nearly constant adsorption at high relative pressure, corresponding to type I isotherms in agreement with the microporous structures of ZIF-8 and ZIF-67 (see Fig. S2 in the ESI). The specific surface areas (1536 and $1574 \mathrm{~m}^{2} \cdot \mathrm{g}^{-1}$ for ZIF-8 and ZIF-67, respectively) are in agreement with previously reported values $[4,9]$.

\subsection{Membrane characterization}

Figs. 3A and $\mathrm{C}$ show a homogeneous monolayer of ZIF-8 and ZIF-67 crystals deposited by dip-coating on the porous PI P84 ${ }^{\circledR}$ support. Fig. S3 in the ESI shows the SEM images used to calculate the percentage of coated area of ZIF-8@PI_1L and ZIF-67@PI_1L treated supports (see Table S1 in the ESI), $99.6 \pm 0.5 \%$ and $59.5 \pm 1.6 \%$, respectively. These different coating yields have to be related to the different particle sizes of the two MOFs [35]. The average particle sizes of the crystals in the continuous layers are $70 \pm 10 \mathrm{~nm}$ for ZIF- 8 and $240 \pm 40 \mathrm{~nm}$ for ZIF-67 (see Fig. S4 in the ESI). Smaller nanoparticles have a larger contact area per unit volume favoring possible interactions between them and with the membrane support surface. ZIF-8@PI_2L and ZIF-67@PI_2L were obtained after depositing by dip coating a second layer of the respective MOFs on top of MOF@PI_1L. For ZIF-8@PI_2L supports (Fig. 3B) the coating area remains at $99.8 \pm 0.1 \%$, within the error comparable to the ZIF-8@PI_1L, while ZIF-67@PI_2L supports (Fig. 3D) exhibit an enhancement in the coating area to $90.7 \pm 6.1 \%$ suggesting that most of the voids generated 
during the first coating were filled (see Fig. S3 from the ESI). EDX mapping in Fig. S5 shows homogeneous distributions of $\mathrm{Zn}$ and Co contents with atomic compositions (Fig. S5E) of $11.2 \pm 0.8 \mathrm{wt} \%$ and $12.5 \pm 0.4 \mathrm{wt} \%$ for ZIF-8@PI_1L and ZIF-8@PI_2L, respectively, and of $18.4 \pm 0.3 w t \%$ and 22.7 \pm 0.9 wt $\%$ for ZIF-67@PI_1L and ZIF-67@PI_2L, respectively. As expected, the content of metal increased when two MOF coatings were carried out. Moreover, the content of $\mathrm{Zn}$ is lower than that of $\mathrm{Co}$, due to the small particle size of ZIF-8 that allowed a higher detection of support because of the penetration of the mapping technique.

The typical ring-like structures of the TFC membranes also appeared in the TFN membranes prepared from the previously coated MOFs@PI substrates (see Figs. 4A, D, E and F). Thus the corresponding top selective PA layers seem to be formed by interfacial polymerization on the typical asymmetrical structure P84 ${ }^{\circledR}$ support (Fig. 4B). The PA/ZIF-8 membrane shows rough surfaces with some protuberances, but there are no interfacial gaps on its surface. It is also observed that the PA and PA/ZIF-8_1L membranes show dense surfaces with no pinholes or nanoparticle agglomerates, indicating that PA was formed correctly on the ZIF-8@PI support (see Fig. 4A). The PA/ZIF-67_1L membrane images (Fig. 4E and F) show that PA was not properly formed on the ZIF-67@PI support. This may be related to the larger particle size of this ZIF-67 which hindered proper PA-PI interaction. The PA/MOF_2L membranes (Figs. 4D and F) were shown to have some bumps with rougher surfaces than the PA/MOF_1L membranes. This suggests that the presence of the second MOF layer in some way hinders the access of the IP reactants to the surface of the PI P84 ${ }^{\circledR}$ substrate, reducing the degree of cross-linking of the selective PA layer and thus leading to slightly different surface morphologies. In fact, it has been reported that a high loading of ZIF-8 can decrease the degree of cross-linking in the PA thin film [7]. 
Moreover, Fig. 4A shows that the surface of the PA/ZIF-8_1L membrane is uniform and almost free of defects. In the cross-section images of this membrane (Figs. 4B and C), the ZIF-8 nanoparticles cannot be clearly distinguished from the dense layer, indicating that the ZIF-8 coating was well integrated with the PA layer because of the good compatibility between ZIF-8 and PA. Fig. 4C shows an image of the cross-section of the PA/ZIF-8_1L membrane where the gray part corresponds to the platinum deposition used during the sample preparation. An amplification of this area (Fig. 4C inset) shows the PA/MOF_1L layer protruding from the PI P84 ${ }^{\circledR}$ support, with a thickness of $70 \pm 10 \mathrm{~nm}$. The SEM images obtained from the upper surfaces of the PA/ZIF-8_2L and PA/ZIF-67_2L membranes are uneven due to the PA formed onto a thicker layer of MOF particles. Figs. S6A, B and C in the ESI shows the places at which EDX compositions (wt\%) were obtained (Table S2 in the ESI), positions 1) and 2) being within the experimental error: 1) detached PA/ZIF-8_2L with 4.1 $\pm 0.8 \mathrm{wt} \%$ of $\mathrm{Zn}$; 2) PA/ZIF-8_2L with $3.6 \pm 1.0 \mathrm{wt} \%$ of $\mathrm{Zn}$; 3) remained PA/ZIF-8_2L with $2.1 \pm 0.4 \mathrm{wt} \%$. This approached the content of $\mathrm{Zn}$ of $1.9 \pm 0.9 \mathrm{wt} \%$ corresponding to PA/ZIF-8_1L (see Fig. S6F). Position 5) in Fig. S6D corresponds to PA/ZIF-67_2L showing an irregular PA surface with $1.3 \mathrm{wt} \%$ of Co from the MOF (see Table S2 of the ESI). Fig. S6E shows an image of the cross-section of the PA/ZIF-67_1L membrane where the PAMOF layer, Pt coating and PI can be distinguished, with a thickness of $240 \pm 40 \mathrm{~nm}$. As expected, these compositions are below those above discussed without the PA coating. In any event, the metal content values confirm the presence of the MOF after the IP process was carried out to obtain the corresponding TFN membranes. Moreover, they demonstrate the suitability of the dip-coating methodology used here to place the MOF particles on top of the PI P84 ${ }^{\circledR}$ support without being eliminated during the IP process. Finally, a comment is due to the coating and membrane thicknesses: as a second coating was performed, the ZIF 
coverage increased, while the coating thickness approximately kept constant. In addition, the PA thickness achieved by interfacial polymerization is particularly small on top of protruding filler particles, what makes that ZIF-8@PI and PA/ZIF were close.

Fig. 5A shows the ATR-FTIR spectra of the cross-linked PI P84 ${ }^{\circledR}$ support, the MOF@PI and the ZIF-8 and ZIF-67 nanoparticles. The peaks at 1348 and $1722 \mathrm{~cm}^{-1}$ in the spectrum of the cross-linked PI $\mathrm{P} 84^{\circledR}$ support correspond to $\mathrm{C}-\mathrm{N}$ and $\mathrm{C}=\mathrm{O}$ bonds, respectively. New characteristic bands of 1300 to $690 \mathrm{~cm}^{-1}$ are observed in the ZIF-8@PI and ZIF-67@PI spectra indicating the presence of the two MOFs in the MOF@PI supports. Fig. 5B corresponds to the ATR-FTIR analysis of the PA and PA/MOF membranes. The PA/MOF membranes show peaks at $1648 \mathrm{~cm}^{-1}(\mathrm{C}=\mathrm{O}$ stretching vibrations of amide), 1535 $\mathrm{cm}^{-1}$ (in-plane $\mathrm{N}-\mathrm{H}$ bending and $\mathrm{C}-\mathrm{N}$ stretching vibrations), and 1467 and $1410 \mathrm{~cm}^{-1}$ (amide functional -NHCO-bond), related to the PA layer formed upon interfacial polymerization [9]. Specifically, in the PA/ZIF-8_2L and PA/ZIF-67_2L TFN membranes, new peaks appear from 690 to $1300 \mathrm{~cm}^{-1}$ due to the presence of the MOFs. Membranes with two MOF coatings were used for this characterization to highlight the presence of ZIF-8 and ZIF-67 in the corresponding TFN membranes.

To study the membrane properties in terms of hydrophilicity / hydrophobicity, the contact angles of the PI, MOF@PI supports and the PA/MOF membranes (see Fig. 6) were measured. PI has a lower contact angle than ZIF-8@PI_2L and ZIF-67@PI_2L, due to the presence of the MOF layer on the porous support, which would increase surface hydrophobicity. On the other hand, the measurements of the contact angles can also be affected by the roughness of the surface favoring the entrapment of air bubbles [36]. Moreover, the porous structure of the support can absorb the superficial water giving rise to relatively low contact angles. The addition of PA to the selective layer allows a more 
impermeable and uniform surface, giving greater hydrophobicity to the membranes. The PA/ZIF-8 and PA/ZIF-67 membranes show higher contact angles than the bare PA membrane, which is again due to the hydrophobic character of ZIFs [4, 26, 27].

The MOF loading on the surface of the PA/ZIF-8_2L and PA/ZIF-67_2L membranes increases, while the PA layer coating on the MOF interlayer tends to be thinner than that of the pure PA membrane. The effect of the hydrophobic MOF layer was therefore more dominant on the contact angle value than the hydrophilic PA, in agreement with the findings of Wang et al. for ZIF-8 [27]. Analogous behavior can be explained for ZIF-67 because of its similar chemical composition to ZIF-8 (both ZIFs share the same ligand) [30].

\subsection{Membrane nanofiltration performance}

Fig. 7 shows that the PA/ZIF-8_1L and PA/ZIF-67_1L membranes give a better performance $\left(8.7 \mathrm{~L} \cdot \mathrm{m}^{-2} \cdot \mathrm{h}^{-1} \cdot \mathrm{bar}^{-1}\right.$ with a $90 \%$ rejection and $\left(4.8 \mathrm{~L} \cdot \mathrm{m}-2 \cdot \mathrm{h}^{-1} \cdot \mathrm{bar}^{-1}\right.$ with a $79.3 \%$ rejection, respectively). In addition, if compared with TFC membranes, the PA/ZIF-8_1L shows a $150 \%$ enhancement in permeance, higher than those achieved with other methods with methanol (see Table 1). On the other hand, Volkov et al. [37] designed dense poly(acrylonitrile) (PAN) membranes modified with poly[1-(trimethylsilyl)-1-propyne] (PTMSP), reporting a MeOH permeance of $7.7 \mathrm{~L} \cdot \mathrm{m}^{-2} \cdot \mathrm{h}^{-1} \cdot \mathrm{bar}^{-1}$ and a retention of $90 \%$ for Remazol Brilliant Blue R $\left(627 \mathrm{~g} \cdot \mathrm{mol}^{-1}\right)$. TFN membranes with carbon nanotubes as fillers have also been used for this purpose, achieving a MeOH permeance of $6.3 \mathrm{~L} \cdot \mathrm{m}^{-2} \cdot \mathrm{h}^{-1} \cdot \mathrm{bar}^{-1}$ and a rejection of $91 \%$ for Brilliant Blue $\mathrm{R}\left(826 \mathrm{~g} \cdot \mathrm{mol}^{-1}\right)$ [38].

The good results obtained with $\mathrm{MeOH}$ by membrane PA/ZIF-8-1L membrane can be also explained by considering the properties of the solvent. The high dielectric constant of $\mathrm{MeOH}$ (32.6) can explain its interaction with the hydrophilic support [39], while the relation 
between the kinetic diameter of $\mathrm{MeOH}(3.6 \AA)$ [40] and the porosity of the sod type topology of both ZIFs with cavity diameters of $11.6 \AA$ accessible through small pore windows with diameters of $3.46 \AA$ (ZIF-8) and $3.43 \AA$ (ZIF-67) [30,41] would suggest some limitation. Nevertheless, we believe that the presence of ZIF-8 brought new transport pathways for smaller $\mathrm{MeOH}$ as compares to the tested dye, Sunset Yellow. In fact, the molecular weight and size of a molecule can also give us information about possible rejection [42]. In this case, Sunset Yellow, with weight $452 \mathrm{~g} \cdot \mathrm{mol}^{-1}$ and an estimated molecular diameter of $1.2 \mathrm{~nm}$ [43], was rejected by our membrane. The rejection was lower for PA/ZIF-67 than for PA/ZIF-8$1 \mathrm{~L}$ due to the presence of defects created by the deficient MOF-polymer contact, as seen above.

Regarding the surface charge influence on the membrane performance, the water isoelectric point is $c a .3 .5$ for the MPD-TMC composite membrane, the zeta potential being $-30.5 \mathrm{mV}$ at $\mathrm{pH} 7.0$ [44]. This suggests no electrostatic attraction with Sunset Yellow (pKa 10.5) in methanol which $\mathrm{pH}$ has to be neutral. This would discard the deprotonation of the dye, facilitating its rejection.

When the number of ZIF coatings was doubled, both membranes PA/ZIF-8_2L (3.6 $\left.\mathrm{L} \cdot \mathrm{m}^{-2} \cdot \mathrm{h}^{-1} \cdot \mathrm{bar}^{-1}\right)$ and PA/ZIF-67_2L $\left(3.0 \mathrm{~L} \cdot \mathrm{m}^{-2} \cdot \mathrm{h}^{-1} \cdot \mathrm{bar}^{-1}\right)$ showed a decline in permeance in comparison with their corresponding PA/MOF_1L and the TFC membranes (see Fig. 7), and only the PA/ZIF-8_2L maintained a high dye rejection of 95\%. This suggests that the two layers of ZIFs tend to accumulate IP reactants giving rise to a thicker PA layer. In consequence, no further increase in the number or ZIF layers was studied considering that permeances below ca. $3.0 \mathrm{~L} \cdot \mathrm{m}^{-2} \cdot \mathrm{h}^{-1} \cdot \mathrm{bar}^{-1}$, far below the performance of the own TFC membrane, would not be desirable. 
The particle size of the filler is an important parameter in TFN membrane preparation as it influences the achievable permeance, as well as the surface properties of the membranes [45]. Table 1 summarizes a selection of methods developed for the preparation of OSN membranes previously reported with other fillers such as ZIF-8, $\mathrm{NH}_{2}-\mathrm{MIL}-53(\mathrm{Al}), \mathrm{MIL}-$ 53(Al), HKUST-1, MIL-47, MIL-101(Cr) and UZM-5, whose particle sizes in general are larger than the nanoparticles used in this work. Figs. S6C and D in the ESI show low thicknesses of the top layers of the PA/MOFs, being $80 \pm 10 \mathrm{~nm}(\mathrm{PA} / \mathrm{ZIF}-8)$ and $244 \pm 50 \mathrm{~nm}$ (PA/ZIF-67), respectively, as compared with those in Table 1.

Unlike the conventional methods of preparing TFN membranes in which the nanoparticles are dispersed in either the aqueous or organic IP phases with poor control of their location (so that most of them may be washed out during the final stages of the TFN membrane preparation), the deposition of MOF nanoparticles by dip-coating on the membrane support allows their location in the TFN film to be controlled. This guarantees a homogeneous and continuous coverage of the support with the filler and allows a more rational use of reactants by avoiding their loss during the membrane washing in the IP process.

Finally, due to the small amount of ZIF material in the TFN [4] XRD was not useful to assess the crystallinity after the IP process, in agreement with similar situations in which small membrane thicknesses of ZIFs presented great difficulties for their direct structural characterization [41], even though some small XRD peaks corresponding to the highest intensities of ZIF-8 and ZIF-67 can be observed in Fig. S7 for the different membrane samples studied here. However, the FTIR observation (Fig. 5) is consistent with the prevalence of the ZIF structure upon the IP process, in agreement with the recent work by Echaide-Górriz et al. where a non-supported thin PA film prepared ad hoc by the IP process 
with the MOF filler embedded was characterized by TEM confirming the presence of MIL101(Cr) [46]. In addition, unsupported PA/MOF composites with a significant higher amount of MOF were prepared without the presence of the P84 ${ }^{\circledR}$ support to corroborate by XRD (see Fig. 8) the preservation of the ZIF-8 and ZIF-67 structures after the IP process and in conditions that are stronger (besides the $10 \mathrm{~min}$ bath, $24 \mathrm{~h}$ under SY-methanol solution) than those used in the OSN experiments carried out here. Fig. 8 confirms than the crystalline of the two MOFs was maintained in the just mentioned conditions.

\section{Conclusions}

A dip-coating method has been developed as an alternative to the conventional method of preparing TFN membranes, in which the location of the MOF (ZIF-8 and ZIF-67) nanoparticles is controlled. This allows a simultaneous decrease in the agglomeration and savings in the use of reactants. The homogeneity and coverage of the monolayer of ZIF deposited on the PI P84 ${ }^{\circledR}$ supports was better with ZIF-8 particles, with the smallest particle size being $70 \pm 10 \mathrm{~nm}$, than with ZIF-67 $(240 \pm 40 \mathrm{~nm}$ in size). The different characterizations confirmed the presence of the crystalline MOFs after the IP process that was carried out to obtain the corresponding TFN membranes. This highlights the suitability of the dip-coating technique used here for placing the MOF particles on top of the PI P84 ${ }^{\circledR}$ support without being eliminated during the IP process. This in turn gave rise to TFN ZIF-8 containing membranes with high methanol permeance (up to $8.7 \mathrm{~L} \cdot \mathrm{m}^{-2} \cdot \mathrm{h}^{-1} \cdot \mathrm{bar}^{-1}$, i.e. an increase of $150 \%$ in comparison with TFC membranes) while maintaining a high SY rejection. However, neither the use of ZIF-67 instead of ZIF-8 nor the application of two dip-coating steps produced an enhancement of the nanofiltration performance of the membranes achieved, probably due to the generation of defects and the creation of a PA layer which was too thick. 
In summary, the dip-coating methodology for controlling the location of MOF nanoparticles in TFN membranes presents an opportunity to minimize the costs and effort associated with their fabrication.

\section{Abbreviations}

ATR attenuated total reflection

BET Brunauer-Emmett-Teller

COF covalent organic frameworks

CR Congo Red

DC Dip-coating

DMF N,N-dimethylformamide

EA ethyl acetate

EDX energy-dispersive X-ray spectroscopy

ETD Everhart-Thornley detector

FEG field emission fun

FIB focused ion beam

FTIR Fourier transform infrared spectroscopy

GS gas separation

HAD hexanediamine

HKUST-1 Hong Kong University of Science and Technology-1

H-MeIm 2-methylimidazolate

IP interfacial polymerization

IPA isopropyl alcohol 
ISA integrally skinned asymmetric

ISG in situ growth

LBL layer-by-layer

$\mathrm{MeOH}$ methanol

MMM mixed matrix membrane

MOF metal-organic framework

MPD m-phenylediamine

NF nanofiltration

OSN organic solvent nanofiltration

PA polyamide

PDMS polydimethylsiloxane

PEG polyethylenglycol

PI polyimide

PP polypropylene

PS polystyrene

PSF polysulfone

PV pervaporation

RB Reactive Black

RO reverse osmosis

SDTA single differential thermal analysis

SEM scanning electron microscopy

SO styrene oligomers

SOD code for the ZIF structure with the same topology than zeolite sodalite

SY Sunset Yellow 
TFC thin film composite

TFN thin film nanocomposite

TGA thermogravimetric analyses

THF tetrahydrofuran

TLD through lens detector

TMC trimesoyl chloride

UV ultraviolet

XRD X-ray diffraction

ZIF $\quad$ zeolite imidazole framework

\section{Acknowledgements}

Financial support from the Spanish Ministry of Economy and Competitiveness (MINECO) and FEDER (MAT2016-77290-R), the European Social Fund (ESF) and the Aragón Government (DGA, T05) is gratefully acknowledged. L.S. is also grateful for a Ph.D. grant from Becas Santander Universidades. The microscopy work was carried out in the Laboratorio de Microscopías Avanzadas at the Instituto de Nanociencia de Aragón (LMAINA, Universidad de Zaragoza). The use of the Servicio General de Apoyo a la InvestigaciónSAI (Universidad de Zaragoza) is also acknowledged.

\section{References}

[1] P. Marchetti, M.F. Jimenez Solomon, G. Szekely, A.G. Livingston, Molecular Separation with Organic Solvent Nanofiltration: A Critical Review, Chem. Rev., 114 (2014) 1073510806. 
[2] M.-B. Wu, Y. Lv, H.-C. Yang, L.-F. Liu, X. Zhang, Z.-K. Xu, Thin film composite membranes combining carbon nanotube intermediate layer and microfiltration support for high nanofiltration performances, J. Membr. Sci., 515 (2016) 238-244.

[3] P. Wen, Y. Chen, X. Hu, B. Cheng, D. Liu, Y. Zhang, S. Nair, Polyamide thin film composite nanofiltration membrane modified with acyl chlorided graphene oxide, J. Membr. Sci., 535 (2017) 208-220.

[4] S. Sorribas, P. Gorgojo, C. Tellez, J. Coronas, A.G. Livingston, High Flux Thin Film Nanocomposite Membranes Based on Metal-Organic Frameworks for Organic Solvent Nanofiltration, J. Am. Chem. Soc., 135 (2013) 15201-15208.

[5] C. Wang, Z. Li, J. Chen, Z. Li, Y. Yin, L. Cao, Y. Zhong, H. Wu, Covalent organic framework modified polyamide nanofiltration membrane with enhanced performance for desalination, J. Membr. Sci, 523 (2017) 273-281.

[6] B. Zornoza, A. Martinez-Joaristi, P. Serra-Crespo, C. Tellez, J. Coronas, J. Gascon, F. Kapteijn, Functionalized flexible MOFs as fillers in mixed matrix membranes for highly selective separation of $\mathrm{CO} 2$ from $\mathrm{CH} 4$ at elevated pressures, Chem. Commun., 47 (2011) 9522-9524.

[7] J.T. Duan, Y.C. Pan, F. Pacheco, E. Litwiller, Z.P. Lai, I. Pinnau, High-performance polyamide thin-film-nanocomposite reverse osmosis membranes containing hydrophobic zeolitic imidazolate framework-8, J. Membr. Sci, 476 (2015) 303-310.

[8] X.L. Dong, Y.S. Lin, Synthesis of an organophilic ZIF-71 membrane for pervaporation solvent separation, Chem. Commun., 49 (2013) 1196-1198.

[9] M.F.J. Solomon, Y. Bhole, A.G. Livingston, High flux membranes for organic solvent nanofiltration (OSN)-Interfacial polymerization with solvent activation, J. Membr. Sci, 423 (2012) 371-382.

[10] J. Campbell, J.D.S. Burgal, G. Szekely, R.P. Davies, D.C. Braddock, A. Livingston, Hybrid polymer/MOF membranes for Organic Solvent Nanofiltration (OSN): Chemical modification and the quest for perfection, J. Membr. Sci, 503 (2016) 166-176.

[11] M. Razali, J.F. Kim, M. Attfield, P.M. Budd, E. Drioli, Y.M. Lee, G. Szekely, Sustainable wastewater treatment and recycling in membrane manufacturing, Green Chem.17 (2015) 5196-5205. 
[12] S. Hermans, H. Mariën, C. Van Goethem, I.F.J. Vankelecom, Recent developments in thin film (nano)composite membranes for solvent resistant nanofiltration, Curr Opin Chem Eng, 8 (2015) 45-54.

[13] L.S. White, Transport properties of a polyimide solvent resistant nanofiltration membrane, J. Membr. Sci, 205 (2002) 191-202.

[14] I. Soroko, M.P. Lopes, A. Livingston, The effect of membrane formation parameters on performance of polyimide membranes for organic solvent nanofiltration (OSN): Part A. Effect of polymer/solvent/non-solvent system choice, J. Membr. Sci, 381 (2011) 152-162.

[15] J.E. Cadotte, EVOLUTION OF COMPOSITE REVERSE-OSMOSIS MEMBRANES, ACS Symp, 269 (1985) 273-294.

[16] P. Vandezande, L.E.M. Gevers, I.F.J. Vankelecom, Solvent resistant nanofiltration: separating on a molecular level, Chem. Soc. Rev., 37 (2008) 365-405.

[17] M. Paul, S.D. Jons, Chemistry and fabrication of polymeric nanofiltration membranes: A review, Polym, 103 (2016) 417-456.

[18] B.-H. Jeong, E.M.V. Hoek, Y. Yan, A. Subramani, X. Huang, G. Hurwitz, A.K. Ghosh, A. Jawor, Interfacial polymerization of thin film nanocomposites: A new concept for reverse osmosis membranes, J. Membr. Sci, 294 (2007) 1-7.

[19] I.-C. Kim, J. Jegal, K.-H. Lee, Effect of aqueous and organic solutions on the performance of polyamide thin-film-composite nanofiltration membranes, J. Polym. Sci., Part B: Polym. Phys, 40 (2002) 2151-2163.

[20] P.B. Kosaraju, K.K. Sirkar, Interfacially polymerized thin film composite membranes on microporous polypropylene supports for solvent-resistant nanofiltration, J. Membr. Sci, 321 (2008) 155-161.

[21] C. Van Goethem, R. Verbeke, S. Hermans, R. Bernstein, I.F.J. Vankelecom, Controlled positioning of MOFs in interfacially polymerized thin-film nanocomposites, J. Mater. Chem. A, 4 (2016) 16368-16376.

[22] L.E.M. Gevers, I.F.J. Vankelecom, P.A. Jacobs, Zeolite filled polydimethylsiloxane (PDMS) as an improved membrane for solvent-resistant nanofiltration (SRNF), Chem. Commun., (2005) 2500-2502.

[23] L.E.M. Gevers, I.F.J. Vankelecom, P.A. Jacobs, Solvent-resistant nanofiltration with filled polydimethylsiloxane (PDMS) membranes, J. Membr. Sci, 278 (2006) 199-204. 
[24] S. Basu, M. Maes, A. Cano-Odena, L. Alaerts, D.E. De Vos, I.F.J. Vankelecom, Solvent resistant nanofiltration (SRNF) membranes based on metal-organic frameworks, J. Membr. Sci, 344 (2009) 190-198.

[25] J. Campbell, G. Szekely, R.P. Davies, D.C. Braddock, A.G. Livingston, Fabrication of hybrid polymer/metal organic framework membranes: mixed matrix membranes versus in situ growth, J. Mater. Chem. A, 2 (2014) 9260-9271.

[26] L. Wang, M. Fang, J. Liu, J. He, L. Deng, J. Li, J. Lei, The influence of dispersed phases on polyamide/ZIF-8 nanofiltration membranes for dye removal from water, RSC Adv, 5 (2015) 50942-50954.

[27] L.Y. Wang, M.Q. Fang, J. Liu, J. He, J.D. Li, J.D. Lei, Layer-by-Layer Fabrication of High-Performance Polyamide/ZIF-8 Nanocomposite Membrane for Nanofiltration Applications, ACS Appl. Mater. Interfaces, 7 (2015) 24082-24093.

[28] C.J. Brinker, G.C. Frye, A.J. Hurd, C.S. Ashley, Fundamentals of sol-gel dip coating, Thin Solid Films, 201 (1991) 97-108.

[29] K.S. Park, Z. Ni, A.P. Côté, J.Y. Choi, R. Huang, F.J. Uribe-Romo, H.K. Chae, M. O'Keeffe, O.M. Yaghi, Exceptional chemical and thermal stability of zeolitic imidazolate frameworks, Proc. Natl. Acad. Sci, 103 (2006) 10186-10191.

[30] R. Banerjee, A. Phan, B. Wang, C. Knobler, H. Furukawa, M. O'Keeffe, O.M. Yaghi, High-Throughput Synthesis of Zeolitic Imidazolate Frameworks and Application to $\mathrm{CO}<\mathrm{sub}>2</$ sub $>$ Capture, Science, 319 (2008) 939-943.

[31] J. Cravillon, S. Münzer, S.-J. Lohmeier, A. Feldhoff, K. Huber, M. Wiebcke, Rapid Room-Temperature Synthesis and Characterization of Nanocrystals of a Prototypical Zeolitic Imidazolate Framework, Chem. Mater, 21 (2009) 1410-1412.

[32] W. Sun, X. Zhai, L. Zhao, Synthesis of ZIF-8 and ZIF-67 nanocrystals with wellcontrollable size distribution through reverse microemulsions, Chem. Eng. J., 289 (2016) 5964.

[33] Y.H. See Toh, F.W. Lim, A.G. Livingston, Polymeric membranes for nanofiltration in polar aprotic solvents, J. Membr. Sci., 301 (2007) 3-10.

[34] F.H. Aragón, P.E.N. de Souza, J.A.H. Coaquira, P. Hidalgo, D. Gouvêa, Spin-glass-like behavior of uncompensated surface spins in NiO nanoparticulated powder, Phys. Rev. B: Condens. Matter, 407 (2012) 2601-2605. 
[35] J. Benito, M. Fenero, S. Sorribas, B. Zornoza, K.J. Msayib, N.B. McKeown, C. Téllez, J. Coronas, Ignacio Gascón, Fabrication of ultrathin films containing the metal organic framework Fe-MIL-88B-NH2 by the Langmuir-Blodgett technique, Colloids Surf., A, 470 (2015) 161-170.

[36] D. Quéré, Rough ideas on wetting, Physica A, 313 (2002) 32-46.

[37] A.V. Volkov, V.V. Parashchuk, D.F. Stamatialis, V.S. Khotimsky, V.V. Volkov, M. Wessling, High permeable PTMSP/PAN composite membranes for solvent nanofiltration, J. Membr. Sci., 333 (2009) 88-93.

[38] S. Roy, S.A. Ntim, S. Mitra, K.K. Sirkar, Facile fabrication of superior nanofiltration membranes from interfacially polymerized CNT-polymer composites, J. Membr. Sci., 375 (2011) 81-87.

[39] X. Yang, A. Livingston, L.F. Dos Santos, Experimental observations of nanofiltration with organic solvents, J. Membr. Sci., 190 (2001) 45-55.

[40] C. Echaide-Górriz, S. Sorribas, C. Téllez, J. Coronas, MOF nanoparticles of MIL-68 (Al), MIL-101 (Cr) and ZIF-11 for thin film nanocomposite organic solvent nanofiltration membranes, RSC Adv., 6 (2016) 90417-90426.

[41] F. Cacho-Bailo, I. Matito-Martos, J. Perez-Carbajo, M. Etxeberría-Benavides, O. Karvan, V. Sebastián, S. Calero, C. Téllez, J. Coronas, On the molecular mechanisms for the $\mathrm{H} 2 / \mathrm{CO} 2$ separation performance of zeolite imidazolate framework two-layered membranes, Chem. Sci., 8 (2017) 325-333.

[42] B. Van der Bruggen, J. Schaep, D. Wilms, C. Vandecasteele, Influence of molecular size, polarity and charge on the retention of organic molecules by nanofiltration, J. Membr. Sci., 156 (1999) 29-41.

[43] H.-S. Park, S.-W. Kang, L. Tortora, Y. Nastishin, D. Finotello, S. Kumar, O.D. Lavrentovich, Self-assembly of lyotropic chromonic liquid crystal sunset yellow and effects of ionic additives, J. Phys. Chem. B., 112 (2008) 16307-16319.

[44] M. Liu, D. Wu, S. Yu, C. Gao, Influence of the polyacyl chloride structure on the reverse osmosis performance, surface properties and chlorine stability of the thin-film composite polyamide membranes, J. Membr. Sci, 326 (2009) 205-214. 
[45] M.L. Lind, A.K. Ghosh, A. Jawor, X. Huang, W. Hou, Y. Yang, E.M.V. Hoek, Influence of Zeolite Crystal Size on Zeolite-Polyamide Thin Film Nanocomposite Membranes, Langmuir, 25 (2009) 10139-10145.

[46] C. Echaide-Górriz, M. Navarro, C. Téllez, J. Coronas, Simultaneous use of MOFs MIL$101(\mathrm{Cr})$ and ZIF-11 in thin film nanocomposite membranes for organic solvent nanofiltration, Dalton Trans., 46 (2017) 6244-6252. 


\section{Figure captions}

Figure 1. Building blocks of: (A) ZIF-8 and (B) ZIF-67. These structures were made with software Diamond 3.2 using the corresponding CIF files [30].

Figure 2. Simulated and experimental XRD patterns of ZIF-8 and ZIF-67. The simulated patterns were obtained using the corresponding CIF files [30].

Figure 3. SEM images of the coated support surfaces: (A) ZIF-8@PI_1L, (B) ZIF-8@PI_2L, (C) ZIF-67@PI_1L and (D) ZIF-67@PI_2L.

Figure 4. SEM images of PA/ZIF-8_1L membrane: (A) surface, (B) cross-section and (C) ion-induced SEM image showing the cross-section membrane using the dual beam technique. SEM images of the membrane surfaces: (D) PA/ZIF-8_2L, (E) (PA/ZIF-67_1L and (F) PA/ZIF-67_2L.

Figure 5. ATR-FTIR spectra after subtracting in case of membranes the PI (P84 $\left.{ }^{\circledR}\right)(\mathrm{A})$ and the PA (TFC) (B) spectra: (A) PI (P84 $\left.{ }^{\circledR}\right)$, ZIF-8@PI_2L, ZIF-67@PI_2L membranes and ZIF-8 and ZIF-67 powders; (B) PA (TFC), PA/ZIF-8_2L, PA/ZIF-67_2L membranes and ZIF-8 and ZIF-67 powders.

Figure 6. Contact angles of the P84, TFN, ZIF-8@PI, ZIF-67@PI, PA/ZIF-8_1L, PA/ZIF8_2L, PA/ZIF-67_1L and PA/ZIF-67_2L membranes.

Figure 7. Effect of solute on OSN using TFC (PA) and TFN membranes: PA/ZIF-8_1L; PA/ZIF-8_2L; PA/ZIF-67_1L and PA/ZIF-67_2L all with DMF bath and DMF filtration post-treatment applied. In case of PA/ZIF-8 membranes, the standard deviations were obtained averaging the results corresponding to 3-4 different membrane samples.

Figure 8. XRD patterns of as prepared PA/ZIF-8 and PA/ZIF-67 unsupported composites and after be submitted to simulated OSN conditions (10 min in DMF and $24 \mathrm{~h}$ in SYmethanol solution). 
Table 1. Different types of methods for the preparation of OSN composite membranes.

\begin{tabular}{|c|c|c|c|c|c|c|c|c|}
\hline Method & $\begin{array}{l}\text { Polymer/ } \\
\text { particle }\end{array}$ & $\begin{array}{c}\text { Particle } \\
\text { size }(\mu \mathrm{m})\end{array}$ & $\begin{array}{c}\text { Thickness of top } \\
\text { layer }(\boldsymbol{\mu m})\end{array}$ & Feed $^{a}$ & $\begin{array}{c}\text { Permeance } \\
\left(\mathbf{L} \cdot \mathbf{m}^{-2} \cdot \mathbf{h}^{-1} \cdot \mathbf{b a r}^{-1}\right)\end{array}$ & $\begin{array}{c}\text { Permeance }^{b} \\
\text { Enhancement } \\
(\%)\end{array}$ & $\begin{array}{c}\text { Rejection } \\
\text { (\%) }\end{array}$ & Ref. \\
\hline $\begin{array}{l}\text { In-situ polymerization } \\
\text { (fillers dispersed in } \\
\text { polymer) }\end{array}$ & PDMS/UZM-5 & $1-1.5$ & $5 \pm 2$ & $\begin{array}{l}\text { WC/ethyl } \\
\text { acetate } \\
\text { WC/toluene }\end{array}$ & 0.6 & NA & $97-98$ & {$[22]$} \\
\hline ISG (in situ growth) & P84 ${ }^{\circledR} /$ HKUST-1 & 16 & $65 \pm 3$ & PS/acetone & $15 \pm 2.7$ & NA & $59-86$ & {$[25]$} \\
\hline \multirow{3}{*}{$\begin{array}{l}\text { Fillers dispersed in } \\
\text { polymer }\end{array}$} & PDMS/ZIF-8 & $0.25-0.5$ & 10 & & 0.5 & NA & 96 & \multirow{4}{*}[24]{} \\
\hline & PDMS/MIL-47 & $1-2$ & 10 & $\mathrm{RB} / \mathrm{IPA}$ & 0.5 & NA & 98 & \\
\hline & PDMS/MIL-53(AI) & $10-15$ & 10 & & 0.5 & NA & 98 & \\
\hline \multirow{3}{*}{$\begin{array}{l}\text { MOF in IP organic } \\
\text { phase }\end{array}$} & PA/ZIF-8 & $0.1 \pm 0.01$ & 0.2 & & $2.5 \pm 0.6$ & 139 & 96 & \\
\hline & $\begin{array}{l}\mathrm{PA} / \mathrm{NH}_{2}-\mathrm{MIL}- \\
53(\mathrm{Al})\end{array}$ & $0.133 \pm 0.013$ & 0.2 & $\mathrm{SO} / \mathrm{MeOH}$ & 1.8 & 128 & 99 & \multirow[t]{2}{*}[4]{} \\
\hline & PA/MIL-101(Cr) & $0.047 \pm 0.006$ & 0.2 & & 3.9 & 233 & 97 & \\
\hline $\begin{array}{l}\text { MOF in both IP } \\
\text { aqueous and organic } \\
\text { phases }\end{array}$ & PA/ZIF-8 & 0.05 & $0.17-0.28$ & CR/water & 2.3 & 202 & 99.9 & {$[26]$} \\
\hline $\begin{array}{l}\text { LBL (layer by layer) } \\
(\mathrm{MOF} \text { on PSF) + IP }\end{array}$ & PA/ZIF-8 & 0.15 & $0.1-0.3$ & CR/water & 2.7 & 179 & 99.2 & {$[27]$} \\
\hline \multirow{2}{*}{ Dip-coating + IP } & $\begin{array}{l}\text { PA/ZIF-8_1L } \\
\text { PA/ZIF-8_2L }\end{array}$ & $0.07 \pm 0.01$ & $\begin{array}{l}0.07 \pm 0.01 \\
0.08 \pm 0.01\end{array}$ & $\mathrm{SY} / \mathrm{MeOH}$ & $\begin{array}{l}8.7 \pm 1.3 \\
3.6 \pm 1.4\end{array}$ & 150 & $\begin{array}{l}90.0 \pm 3.9 \\
94.5 \pm 3.8\end{array}$ & \multirow[t]{2}{*}{$\begin{array}{l}\text { This } \\
\text { work }\end{array}$} \\
\hline & $\begin{array}{l}\text { PA/ZIF-67_1L } \\
\text { PA/ZIF-67_2L }\end{array}$ & $0.24 \pm 0.04$ & $\begin{array}{l}0.240 \pm 0.04 \\
0.244 \pm 0.05\end{array}$ & $\mathrm{SY} / \mathrm{MeOH}$ & $\begin{array}{l}4.8 \\
3.0\end{array}$ & & $\begin{array}{l}79.3 \\
88.2\end{array}$ & \\
\hline
\end{tabular}

${ }^{a} \mathrm{~W}$ : Wilkinson catalyst $925 \mathrm{~g} \cdot \mathrm{mol}^{-1}$; SO: styrene oligomers $236 \mathrm{~g} \cdot \mathrm{mol}^{-1}$; CR: Congo Red $697 \mathrm{~g} \cdot \mathrm{mol}^{-1}$; RB: Reactive Black $1017 \mathrm{~g} \cdot \mathrm{mol}^{-1}$; SY: Sunset Yellow $452,4 \mathrm{~g} \cdot \mathrm{mol}^{-1}$; EA: ethyl acetate THF: tetrahydrofuran, PS: styrene oligomers $450 \mathrm{~g} \cdot \mathrm{mol}^{-1}-1500 \mathrm{~g} \cdot \mathrm{mol}^{-1} .{ }^{b} \mathrm{The}$ permeance enhancement was calculated from the permeance ratio of the TFN membrane to the TFC membrane. 
Figure 1

A

B

\section{ZIF-8}

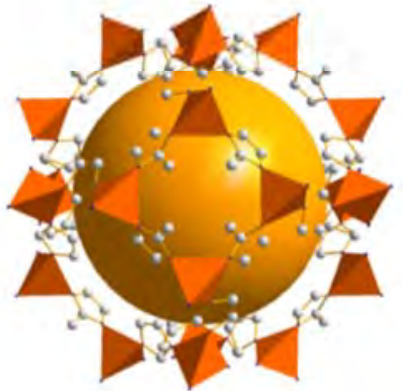

ZIF-67

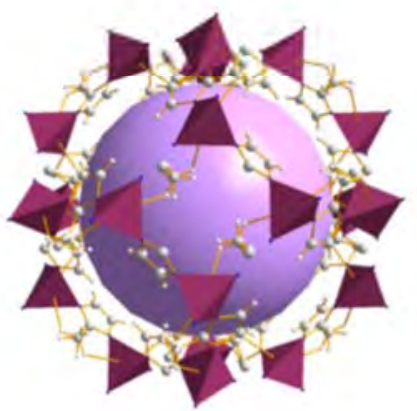


Figure 2

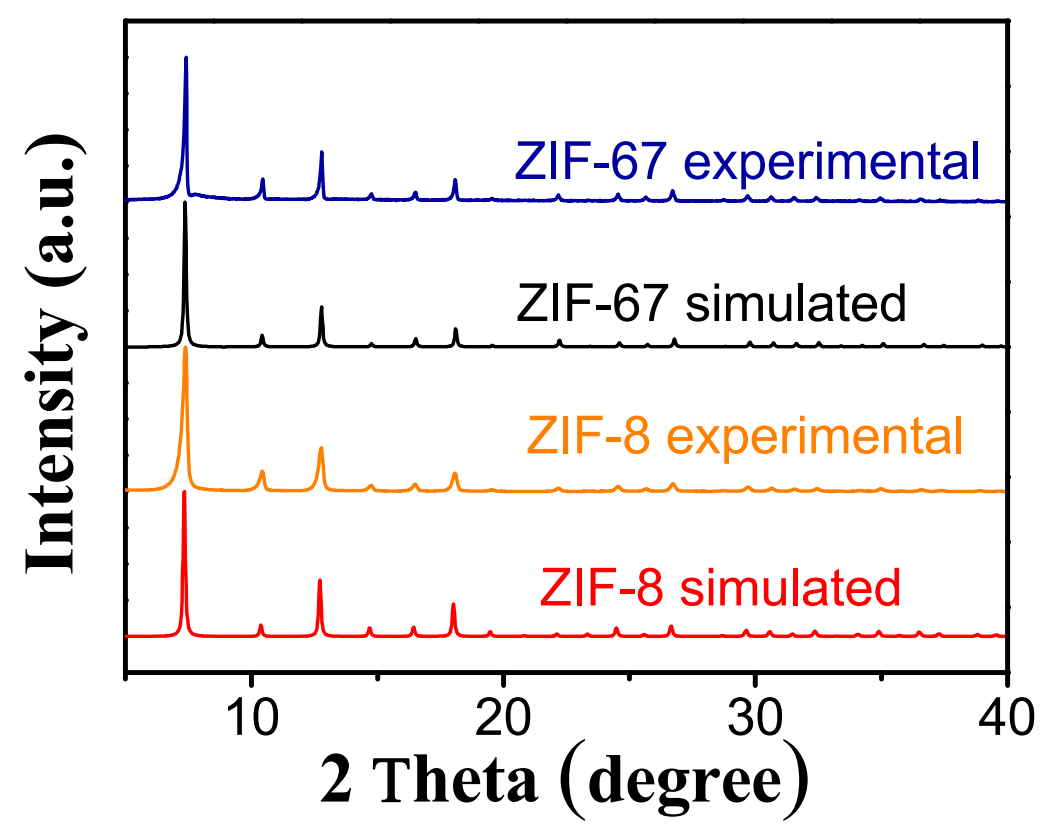


Figure 3
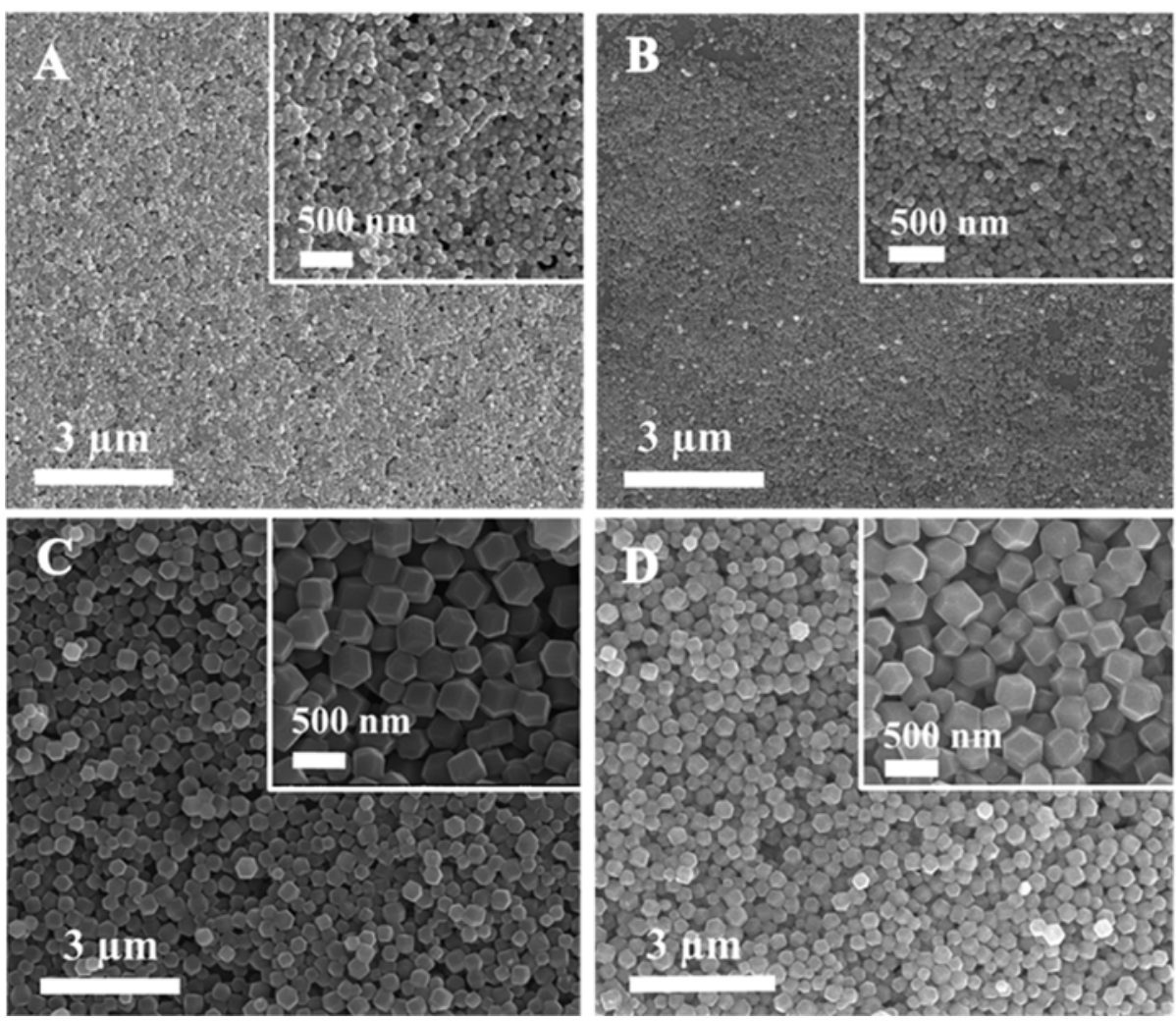
Figure 4

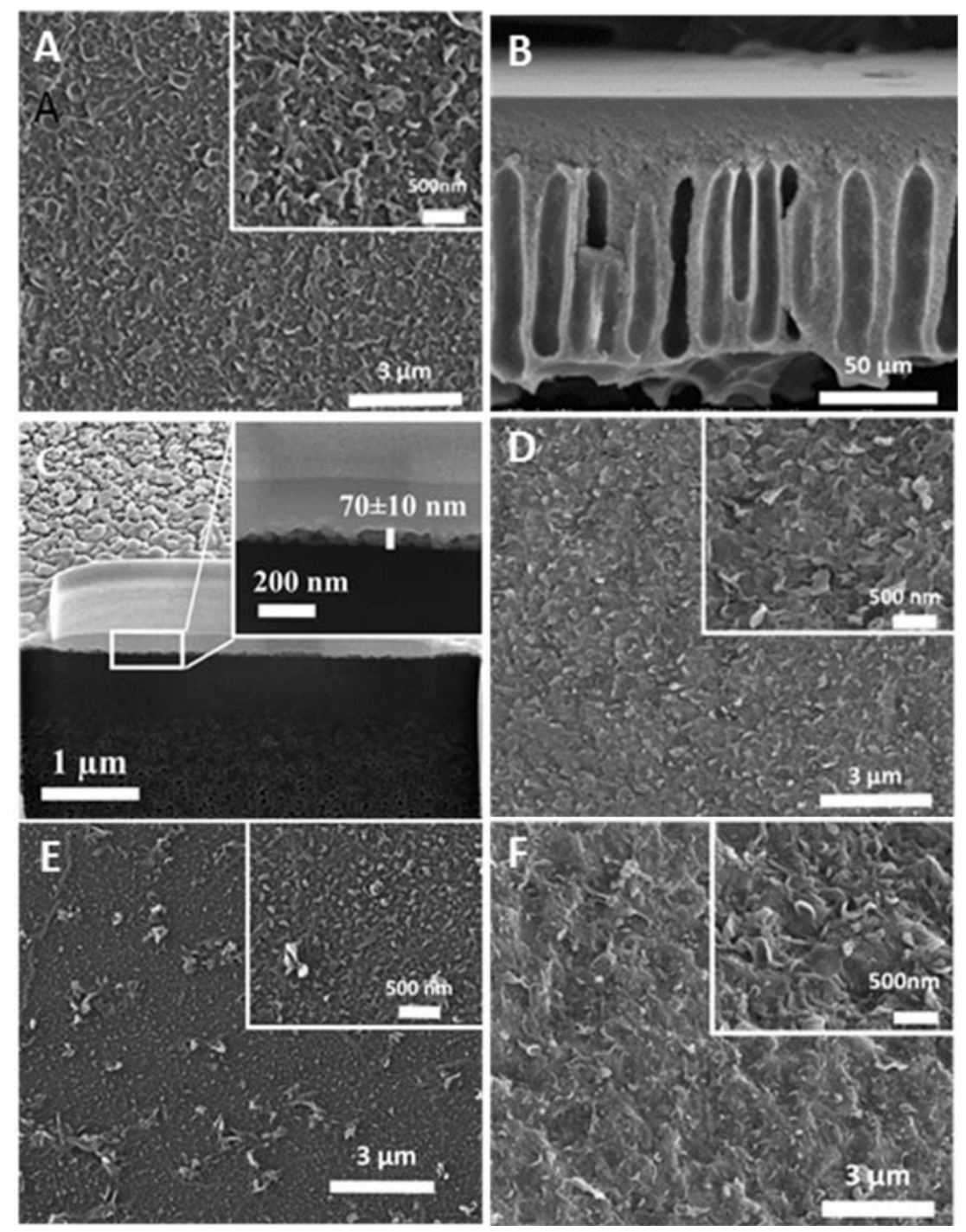


Figure 5
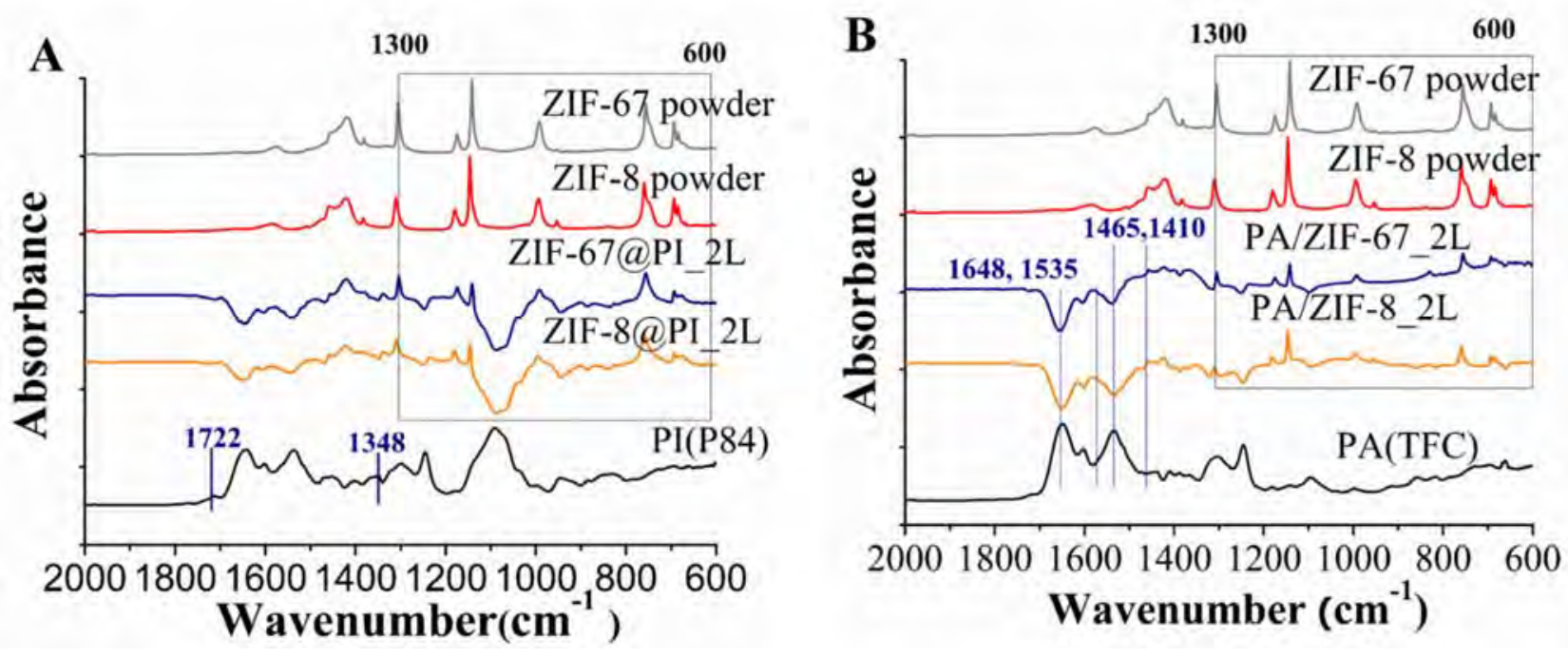
Figure 6

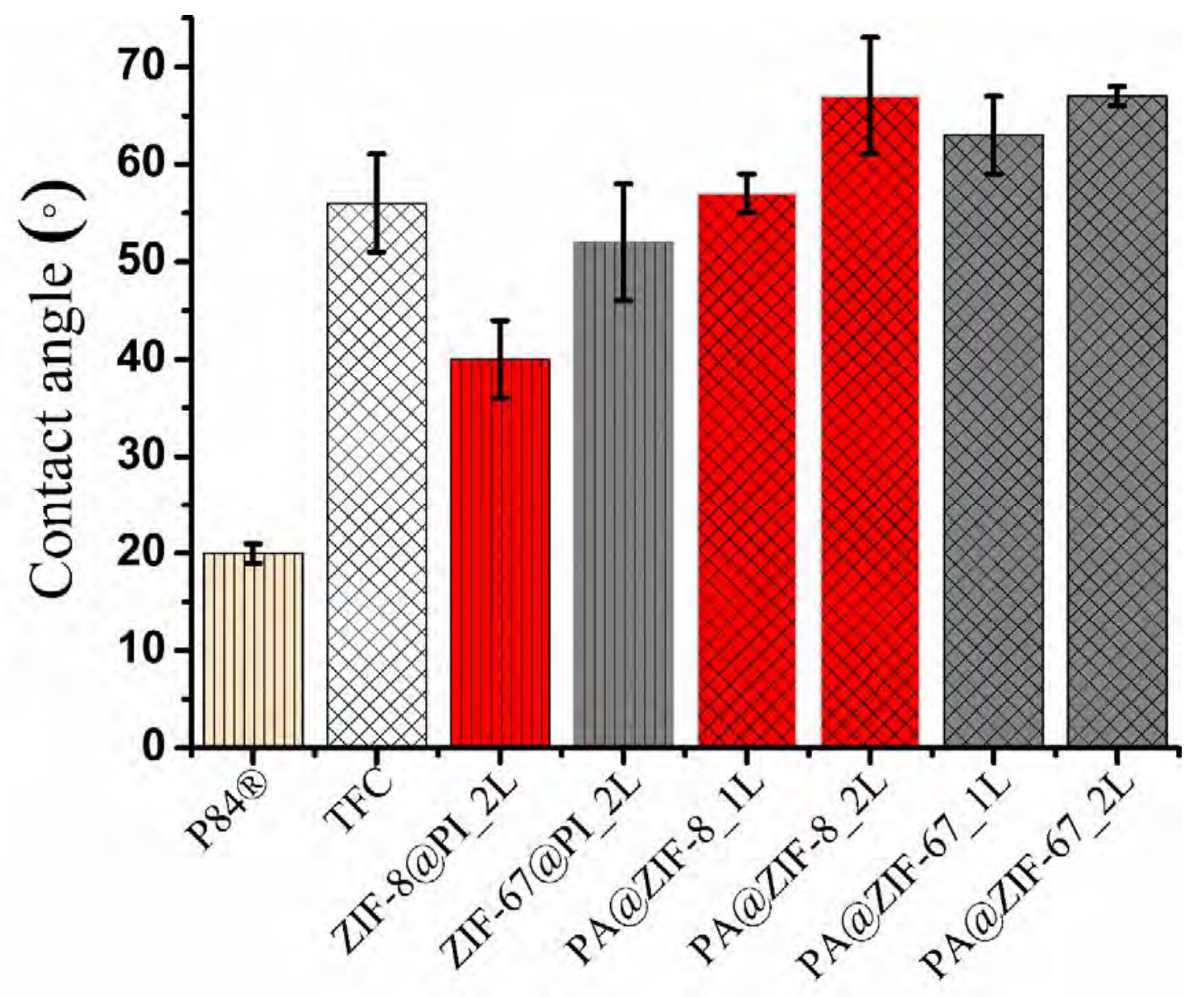


Figure 7

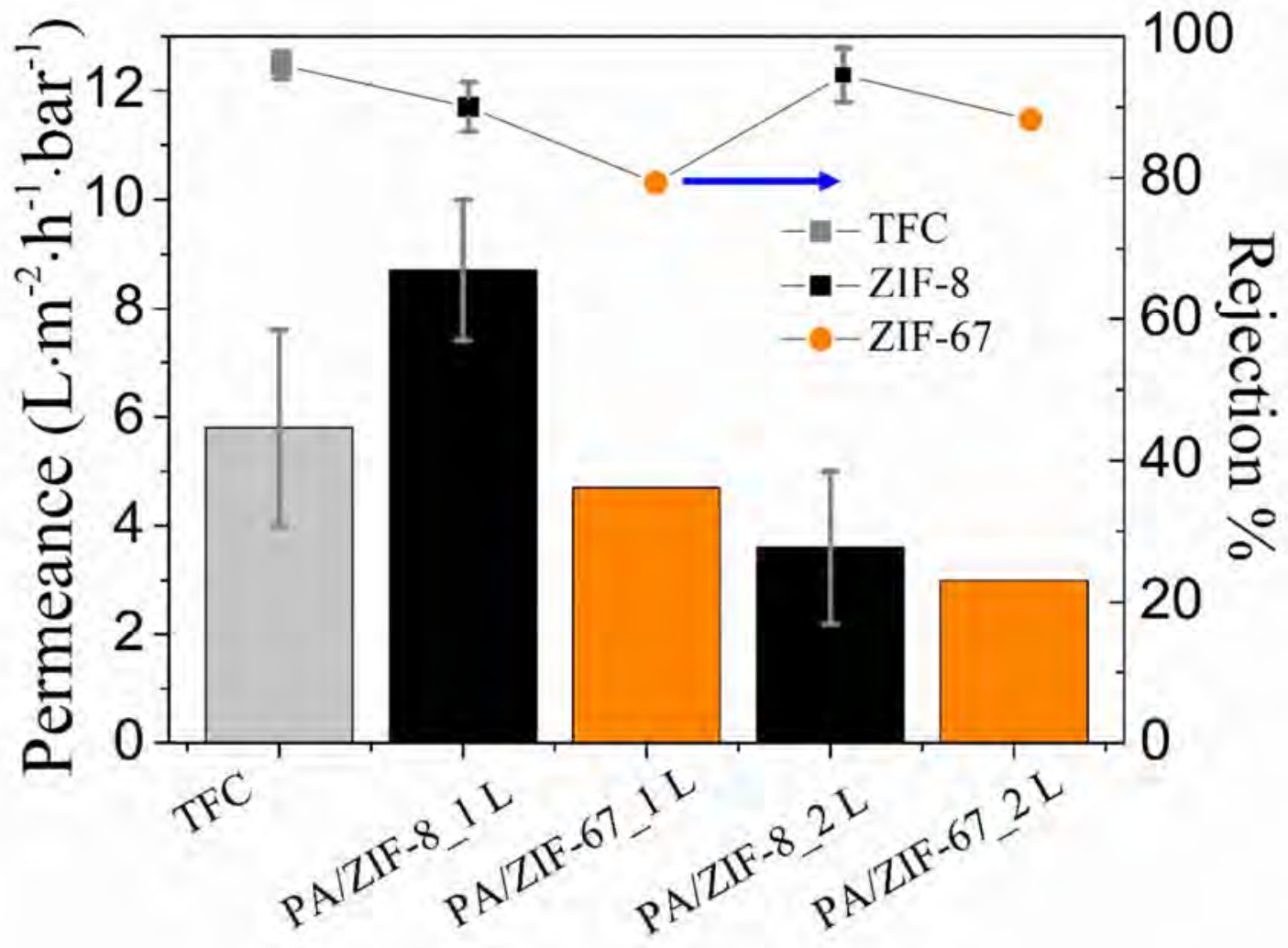


Figure 8

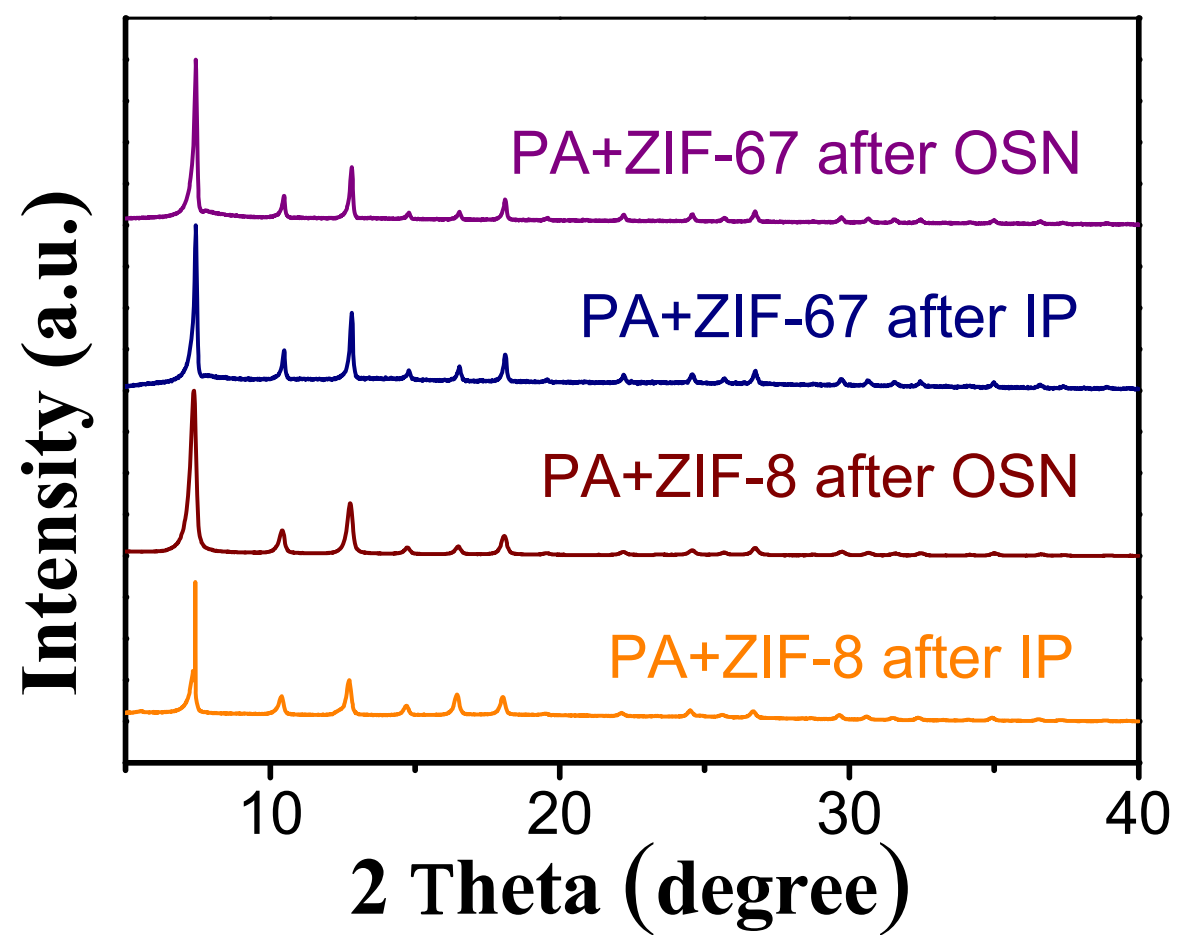

